\title{
FEATURE SELECTION AND CLASSIFICATION OF INDIAN MUSICAL STRING INSTRUMENTS USING SVM
}

\author{
Seema R. Chaudhary \\ Associate Prof, Dept. of Computer Science \& Engineering, \\ Maharashtra Institute of Technology, Aurangabad \\ Maharashtra, India \\ srchary03@gmail.com \\ Sangeeta N. Kakarwal \\ Associate Professor, Dept. of Computer Science \& Engineering \\ PES College of Engineering, Aurangabad \\ Maharashtra, India \\ s_kakarwal@yahoo.com \\ Jayashri V. Bagade \\ Associate Prof., Dept. of Information Technology \\ Vishwakarma Institute of Information Technology, Pune \\ Maharashtra, India \\ jayashrihedaoo@rediffmail.com
}

\begin{abstract}
Music is one of the most important aspects of human life. It touches your soul and relaxes your mind and body. Many musicians from all over the world share their music on social media platforms. On the internet, you can listen to a wide range of music. It is difficult and time-consuming to search for a specific one based on the singer, genre, music piece, instrument, and so on. Previous attempts were mostly concerned with categorising various instruments belonging to different families, such as woodwind, brass, string, percussion, and so on. The objective of this paper is to categorise musical instruments of the string family, particularly Indian musical instruments of the strucked and plucked types. Experimentation is carried out using monophonic recordings of solo instrument artists. Temporal, spectral, statistical, and the first 13 Melfrequency cepstral coefficients have all been considered in audio features. Support Vector Machine (SVM) with linear, radial basis, and quadratic kernels was used for classification. The first two experiments filter features using MANOVA and the Chi-square method. In the third experiment, temporal, spectral, statistical, and MFCC features are chosen for classification independently. The highest accuracy, 93.4 percent, was obtained by using the top five selected features. For selected features, there is only a $+1 \%$ variation in accuracy when using SVM with different kernels. For MFCC 13 features, the linear kernel had an accuracy of 92.5 percent, while the radial basis kernel had an accuracy of 52.6 percent.
\end{abstract}

Keywords: Musical Instruments, classification, temporal, spectral, MFCC, Feature selection, Chi-square, Support Vector Machine

\section{Introduction}

Musical information retrieval(MIR) is one of the emerging research areas. It includes features such as artist recognition, song searching and sorting, music genre classification, music synthesis, and musical instrument recognition. Musical instruments are an essential component of music. Each instrument has its own sound colour due to the material it is made of, size, shape, and so on. Percussion, wind, string, brass, and electronic instruments are the four types of musical instruments. String instruments are further classified according to their playing style, which includes strucked, plucked, and bowed instruments. The majority of MIR research has focused on western 
musical instruments. Few people have worked on Indian musical instruments. Those who have worked have classified Indian instruments into various families.

In this paper, we focused on the string family, with a particular emphasis on Indian musical string instruments such as the Santoor, Sitar, Sarod, and Veena. Santoor plays in a struck style, whereas Sitar, Veena, Sarod, and Guitar are plucked. Guitar is also included in the dataset because it is a popular string instrument. Audio features are analysed for the instruments, feature selection using a filter approach is investigated, and then Support Vector Machine (SVM) using linear, radial basis, and quadratic kernels is classified.

The remaining paper is divided into four sections, with a review of related work in Section 2. Section 3 discussed the methodology we proposed for classifying musical string instruments. The results of experimentation are discussed in Section 4. Section 5 contains the conclusion.

\section{Related Work}

The method used to classify wind and string family instruments was SVM. SVM 's c parameter used to optimise feature selection [Ashwini and Vijaya (2020) ]. It is also investigated using K Nearest Neighbor (KNN) algorithms [Chandan (2019)]. KNN and a Feedforward backpropagation neural network (FFBNN) classifiers[Joshi and Chitre (2015)] used to recognise flute, harmonium, and sitar instruments. FFBNN for linear predictive coefficients has achieved high accuracy. In order to extract temporal features from Sarod concerts [Vinutha et al. (2016)], effective onset detection methods were developed. Indian musical instruments [ Kumar et al. (2020)] classified with speech. To distinguish Carnatic music instruments, the MFCC and LPC characteristics are used [Shetty and Hegde (2020)]. Accuracy using MFCC and Artificial Neural Network classifier performs better than LPC and Bayesian classifier, SVM, also analyzed impact of frame sizes on accuracy.

Sound signal is segmented into non overlapping frames. Co-occurrence matrix of Pearson's correlation coefficients computed for all frames. Features are calculated from computed matrix to classify speeches and songs based on their periodicity[ Ghosal et al.(2020)]. With the use of Chroma-Reduced Pitch (CRP) features and Mel Frequency Cepstral Coefficient (MFCC) features, [Sangeetha and Nalini (2020)] singer identified by SVM classifier. SVM with MFCC $92.0 \%$ accuracy, CRP $44.0 \%$ and combined MFCC and CRP $94.0 \%$ accuracy achieved. The proposed a model [ Joder et al. (2009)] based on Sonic Unit segmentation, partitions the signal based on musical notes and temporal integration (Early and late integration). Mean/variance flaws reduced classification complexity but did not improve performance during early integration. SVM with sonic unit outperformed HMM and GMM in late integration. When early and late integration are combined, classification improves significantly.

Long-term and short-term timbral features and an SVM classifier used to identify content-based music genres. Timbral characteristics such as MFCC and log energy with varying frame lengths were extracted. The linear kernel achieved $86.4 \%$ accuracy, the exponential radial basis kernel 88.3 percent accuracy, and the polynomial kernel 94.8\% accuracy[Chen and Chen (2009)]. Classification results improved when polyphonic audio is separated into streams during preprocessing [Bosch et al. (2012)].

Experiments have used segregation methods such as A Flexible Audio Source Separation Framework, which was developed based on simple Right-Mid/Left/Side panning information(Ozerov et al. (2011)]

Audio was segmented into frames and then frames were classified using multiclass SVM using LPCC,MFCC, and ICA-based features [Elaiyaraja and Sundaram (2012)]. Audio has been classified based on classification of frames. In this paper [Kothe et al.(2016)] explored 31 features with SVM and KNN classifiers in this paper, which include temporal, spectral, perceptual, and wavelet-based features. It was discovered that after using the features selection technique to optimise features, classification performance improved. The highest 90 percent accuracy for spectral roll off with roughness and spectral centroid was reached using the k-NN classifier to classify musical string instruments( Kakarwal ans Chaudhary (2020)]

It was observed (Rachrela et al. (2020)] that the spectrogram for the same note played on different instruments differs. The MFCC and spectral features are extracted, and supervise methods and unsupervised machine learning algorithms -Hierarchical Clustering - are used. SVM outperformed the other methods.

Most of these studies have done research on the classification of western musical instruments. Few people have explored Indian musical instruments. The majority of the work done in Indian musical instrument classification has classified instruments that belong to different families. In this paper, we focused on the classification of Indian musical instruments from the string family using supervised learning. Finding appropriate features for musical instruments is difficult. We present feature selection using a filter approach by statistical methods in this paper.

\section{Proposed Methodology}

Figure 1 depicts the proposed system, which is described in the following steps.

Step 1: Input - As an input, audio samples in.wav format are provided.

Step 2: Preprocess - Silent samples from audio files are removed from the beginning and end of the signal.

Step 3: Extraction of Audio Features - A total of 30 audio features are extracted from preprocessed files. 
Step 4: Normalization - Max - Min scaling is used to normalise all values between 0 and 1.

Step 5: Feature Selection - To select significant features, the statistical method MANOVA is used.

Step 6: Training $-75 \%$ of samples were used to train the model.

Step 7: Classify - A SVM classifier is used to classify $25 \%$ of the testing samples.

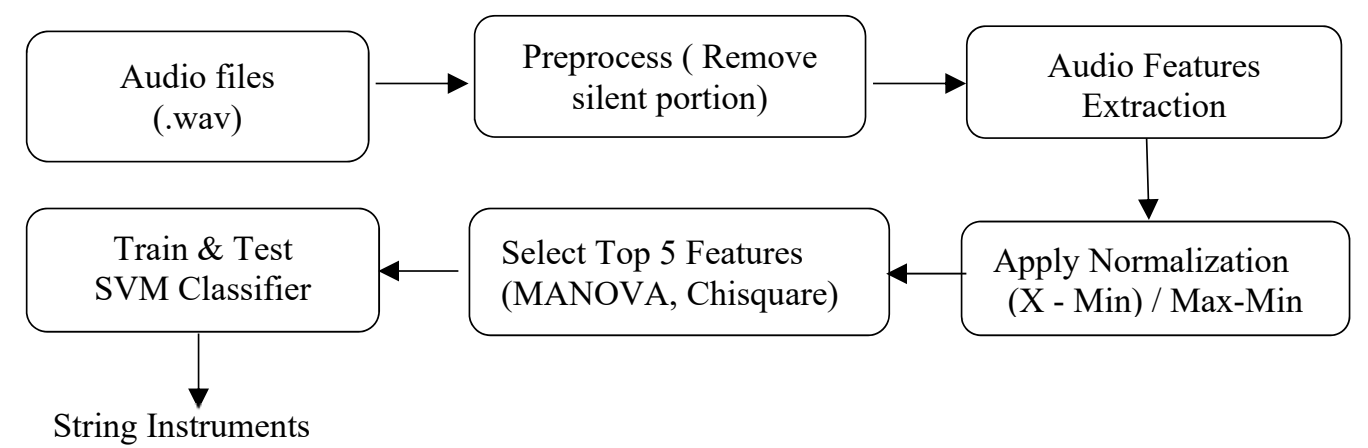

Fig. 1. Block Diagram of Proposed System

\subsection{Dataset}

The database was collected using monophonic recordings of Indian musical string instruments such as the Santoor, Sarod, Sitar, Veena, and Guitar. MP3 files are converted to wave format with a sampling rate of 44.1 $\mathrm{KHz}$. The dataset contains a total of 250 samples. Each musical string instrument has received 50 audio samples. Each sample is 5 seconds long. The data is preprocessed by removing the silent portion from the beginning and end of each sample.

\subsection{Feature Extraction}

Audio features are highly important in audio classification. The following features[Peetersa et al. (2011)] are being considered for experimentation.

- Zero crossing rate $(\mathrm{Zc})$ - It counts the number of times a signal waveform crosses the zero amplitude line during a given time period as a result of a transition from a positive/negative to a negative/ positive value.

$$
Z c_{t}=\frac{1}{N} \sum_{k}^{n} \operatorname{sign}(y[n])-\operatorname{sign}(y[n-1])
$$

- Spectral centroid(Sc) is useful find geometric center (centroid) of the distribution.

$$
\mu 1=\int y \cdot \rho(y) \cdot \delta y
$$

Where y are the observed data and $\rho(y)$ is the probability to observe

- Spectral spread(Ss) calculates standard deviation of the data.

$$
\sigma^{2}=\mu_{2}=\int\left(y-\mu_{1}\right)^{2} \cdot \rho(y) \cdot \delta y
$$

- Spectral rolloff( $\mathrm{Sr})$ calculates the amount of high frequency in a signal by determining the frequency at which a certain fraction of total energy is contained below a given frequency

$$
\sum_{0}^{f q} a^{2}(f)=0.85 \sum_{0}^{\frac{N y}{2}} a^{2}(f)
$$

Where fq is spectral roll off frequency and $\mathrm{Ny} / 2$ Nyquist frequency

- Kurtosis gives the (excess) kurtosis, of the data.It gives measure of the flatness of a distribution around its mean value.It is computed from the 4 th order moment.

$$
\gamma_{2}=\int(y-\mu)^{4} \cdot \rho(y) \cdot \delta y / \sigma^{4}
$$

- Brightness $(\mathrm{Br})$ measures the amount of energy above the given frequency. 
- Roughness $(\mathrm{Rg})$ computes the spectrum's peaks and takes the average of all dissonance between all possible pairs of peaks.

- Irregularity(Ir) gives degree of variation of the successive peaks of the spectrum.

- The flatness(Fl) indicates whether the distribution is smooth or spiky. It calculates ratio between the geometric mean and the arithmetic mean given by Eq.6.

$$
F l=\frac{\sqrt[N]{\prod_{n=0}^{N-1} y(n)}}{\left(\frac{\sum_{n=0}^{N-1} y(n)}{N}\right)}
$$

- Mel-frequency cepstral coefficients (MFCC) represent the spectral shape of a sound with a small number of cooeficients. The cepstrum is the Fourier Transform of the spectrum's logarithm. The Mel-cepstrum is the cepstrum computed using Mel-bands rather than the Fourier spectrum shown in Figure 2.First 13 features of MFCC have been considered for experiment.

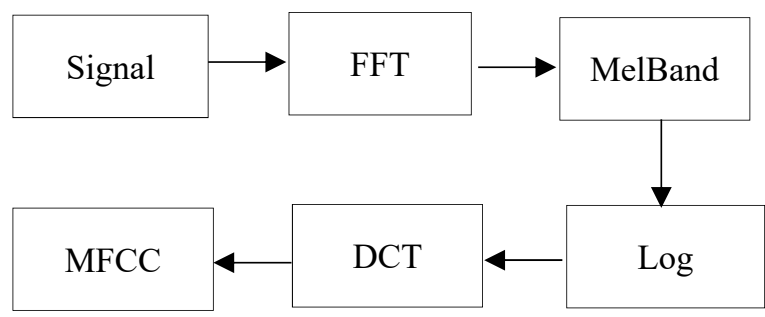

Fig. 2. MFCC formulation

Statistical information is also extracted like min, max, mean, standard deviation, dynamic range, entropy, crest factor included in feature set.

\subsection{Feature Selection}

One of the most important steps before classification is feature selection. Filter methods [Bommert et al. (2020)] are useful for reducing the number of features in a small dataset.The filter approach was used to select features. Two methods are used to filter out the least significant features and select the most prominent ones: multivariate analysis of variance (MANOVA) and chi-squared test [McHugh (2013)] features. The top five distinguishing features have been identified.

\section{- One-way Multivariate analysis of variance (MANOVA)}

It is used to measure difference between one continuous dependent variable and one independent grouping variable. The $\mathrm{F}$ value and $\mathrm{p}$ scores are computed. The $\mathrm{F}$ value measure defines whether or not the means of different samples differ significantly.

Sum of squares between group $\left(S S_{\text {betwween }}\right)$ is calculated as

$$
S S_{\text {betwween }}=\sum_{i=1}^{k} n_{k}\left(\bar{X}_{l}-\bar{X}_{G}\right)^{2}
$$

where $\mathrm{X}_{\mathrm{i}}$ is sample means and $\mathrm{X}_{\mathrm{G}}$ is grand mean

Mean Squares between group $\left(M S_{\text {betwween }}\right)$ is calculated by

where $\mathrm{k}-1$ is degree of freedom

$$
M S_{\text {betwween }}=S S_{\text {betwween }} / k-1
$$

Sum of squares within group ( $\left.S S_{\text {within }}\right)$ is calculated by Eq.(9)

$$
S S_{\text {within }}=\sum\left(X_{i j}-\bar{X}_{j}\right)^{2}
$$

Mean Squares within group $\left(M S_{\text {within }}\right)$ is calculated by

$$
M S_{\text {withinn }}=S S_{\text {within }} / N-k
$$

where $\mathrm{N}-\mathrm{k}$ is degree of freedom $\mathrm{N}=$ sum of the sample sizes $\mathrm{k}=$ number of samples 


$$
\text { F value }=M S_{\text {between }} / M S_{\text {within }}
$$

Features whose $\mathrm{F}$ value is high and p-value less than 0.05 are given importance .

\section{- $\quad$ Chi-squared test}

The chi-squared test calculates scores for each feature which gives how much difference exists between your observed output and the actual output given in below Equation 12. A low value for chi-square means there is a high correlation between feature and output.

$$
x_{d}^{2}=\sum\left(O b s_{i}-\operatorname{Exp}_{i}\right)^{2} / \operatorname{Exp}_{i}
$$

where $\mathrm{d}$ is degree of freedom, Obs is observed output and Exp is expected output.

\subsection{SVM Classification}

The goal of SVM is to separate data points by hyper plane and using kernel trick extends nonlinear boundaries of hyper plane. Accordingly SVM, find support vectors i.e. points that are close to both the classes. In the next step, find the nearness of support vectors with dividing plane. The margin is the distance between the points and the dividing line. The purpose of an SVM algorithm is to maximize margin[Liu et al. (2010)]. Maximum margin means hyper plane is optimal. Selection of proper kernel enhances the performance of SVM.

Kernel transforms input data into high dimensional feature space. In the new feature space data is easily separable. There are different types of kernel functions are available. In this paper, experimentation has been carried out using linear, radial basis function (RBF) and quadratic(Quad) kernels.

Linear kernel : It is the most basic type of kernel, usually one dimensional in nature.

Where $x_{i}, x_{j}$ are data points and . is dot product

$$
F\left(x_{i}, x_{j}\right)=\sum x_{i} \cdot x_{j}
$$

\section{Gaussian Radial Basis Function (RBF) :}

$$
F\left(x_{i}, x_{j}\right)=\exp \left(-\operatorname{gamma}\left\|\left(x_{i}-x_{j}\right)\right\|^{2}\right)
$$

The value of gamma varies from 0 to 1.

\section{Quadratic Function :}

$$
F\left(x_{i}, x_{j}\right)=\left(x_{i} \cdot x_{j}+1\right)^{2}
$$

Multi class classification is accomplished by one versus one approach in SVM.

\section{Experiments and Results}

\subsection{Experimental setup}

Database composed of monophonic recordings of Indian string instruments. The majority of the recordings are from commercial CDs. Each sample is 5 seconds long. Pre - processing stage removes the silence at the beginning and end of the audio. Audio features are extracted based on their temporal, spectral, and statistical properties. Max-Min normalisation is used to normalise features. In the case of negative values, the absolute of the minimum negative value is added to each value of that feature, and then Min-Max normalisation is applied.

MANOVA and the Chi-Square test are used to identify significant features, which are then classified using SVM. The method of 5 fold cross validation is used. The model is trained with $70 \%$ of the samples and tested with $30 \%$ of the samples.

\section{- Experiment 1 : Feature Selection using One-way MANOVA method}

To identify the top five prominent features, the one-way -MANOVA [Steyn and Ellis (2009)] method is used. Features with a high $F$ value and a low $p$ value are chosen. Table 1 and Table 2 show the statistics for the top five selected features: The highest $\mathrm{F}$ value was obtained for the mean feature. In the first five, only $4^{\text {th }}$ and $8^{\text {th }}$ coefficient of MFCC got selected. 


\begin{tabular}{|l|l|r|r|}
\hline \multicolumn{1}{|c|}{ Top 5 Features } & \multicolumn{1}{c|}{ Type } & F & P value \\
\hline Mean & Statistical & 981.0082 & $1.9 \mathrm{E}-149$ \\
\hline Flatness(Fl) & Spectral & 279.0453 & $5.95 \mathrm{E}-90$ \\
\hline MFCC-4 ${ }^{\text {th }}$ Coefficient(M4) & MFCC & 271.8336 & $8.16 \mathrm{E}-89$ \\
\hline MFCC- $8^{\text {th }}$ Coefficient(M8) & MFCC & 182.3868 & $3.25 \mathrm{E}-72$ \\
\hline Standard Deviation(Std) & Statistical & 176.3588 & $6.93 \mathrm{E}-71$ \\
\hline
\end{tabular}

Table 1. One-way MANOVA-Top 5 Features with MFCC

\begin{tabular}{|l|l|r|r|}
\hline \multicolumn{1}{|c|}{ Top 5 Features } & \multicolumn{1}{c|}{ Type } & $\boldsymbol{F}$ & P value \\
\hline Mean & Statistical & 981.0082 & $1.9 \mathrm{E}-149$ \\
\hline Flatness & Spectral & 279.0453 & $5.95 \mathrm{E}-90$ \\
\hline Standard Deviation & Statistical & 176.3588 & $6.93 \mathrm{E}-71$ \\
\hline Spectral Spread(Ss) & Spectral & 166.1547 & $1.48 \mathrm{E}-68$ \\
\hline Spectral Rolloff(Sr) & Spectral & 162.5816 & $1.02 \mathrm{E}-67$ \\
\hline
\end{tabular}

Table 2. One-way MANOVA-Top 5 Features without MFCC

After forming a vector of the top five features and training an SVM classifier model, new samples are classified. Figure 3 represents the average accuracy obtained using selected features.

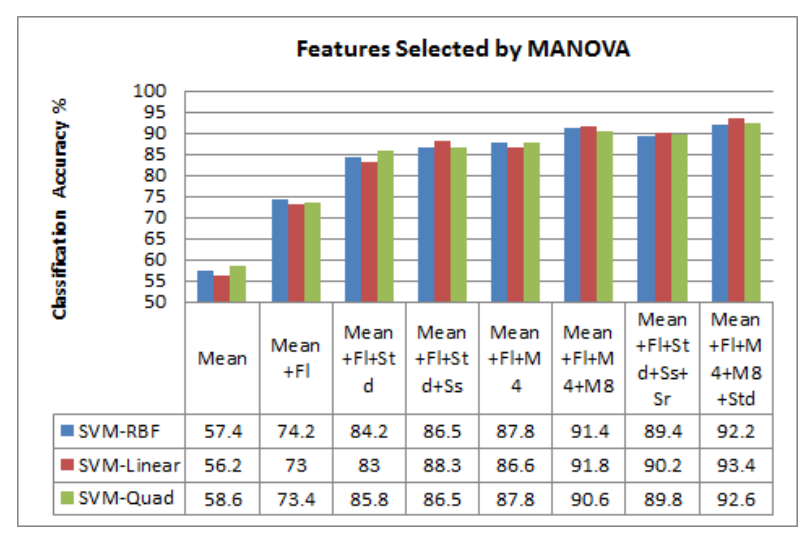

Fig. 3. SVM Average Accuracy(\%) for MANOVA-Selected Features

\section{- Experiment 2: Feature Selection using Chi-square test}

Tables 3 and Table 4 show the top five features based on the Chi-square test. Among the first five features, the $4^{\text {th }}$ coefficients of MFCC (out of 13 coefficients) are chosen using Chi-square.

\begin{tabular}{|l|c|l|}
\hline \multicolumn{1}{|c|}{ Top 5 Features } & Abbreviation & \multicolumn{1}{c|}{ Type } \\
\hline Mean & Mean & Statistical \\
\hline Flatness & $\mathrm{Fl}$ & Spectral \\
\hline Zero crossing rate & $\mathrm{Zc}$ & Time \\
\hline MFCC- $4^{\text {th }}$ Coefficient & $\mathrm{M} 4$ & MFCC \\
\hline Spectral Rolloff & $\mathrm{Sr}$ & Spectral \\
\hline
\end{tabular}

Table 3. Chi-square-Top 5 Features with MFCC 


\begin{tabular}{|l|c|l|}
\hline \multicolumn{1}{|c|}{ Feature } & Abbreviation & \multicolumn{1}{c|}{ Type } \\
\hline Mean & Mean & Statistical \\
\hline Flatness & Fl & Spectral \\
\hline Zero crossing rate & $\mathrm{Zc}$ & Time \\
\hline Spectral Rolloff & $\mathrm{Sr}$ & Spectral \\
\hline Standard Deviation & $\mathrm{Std}$ & Statistical \\
\hline \multicolumn{2}{|c|}{ Table 4. Chi-square-Top 5 Features without MFCC } \\
\hline
\end{tabular}

The average accuracy obtained by SVM classifiers using different kernels for Chi-square selected features is shown in Figure 4.

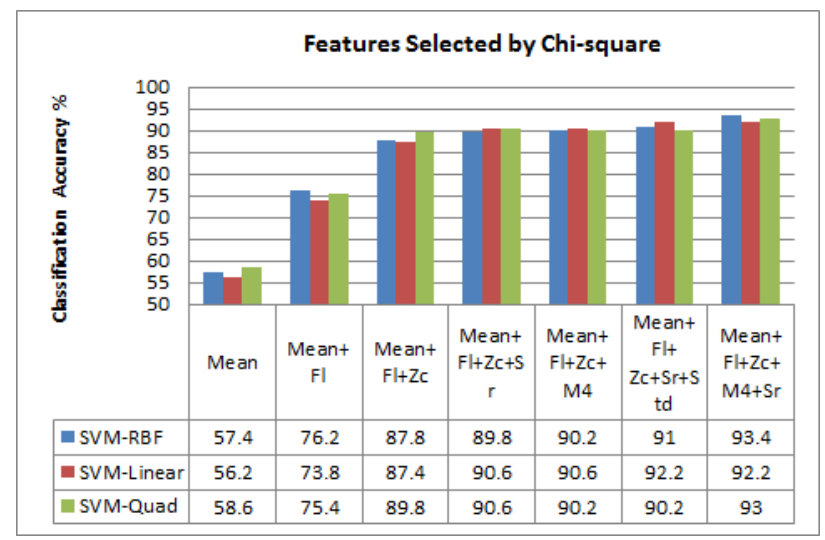

Fig. 4. SVM average accuracy(\%) for Chi-square selected features

\section{- Experiment 3: Feature Selection using Category}

In this experiment, we have explored features by their type. A set of features is grouped by type (as shown in table 5) and then given to SVM classifier.

\begin{tabular}{|l|c|l|}
\hline \multicolumn{1}{|c|}{ Type } & $\begin{array}{c}\text { No. of } \\
\text { features }\end{array}$ & \multicolumn{1}{c|}{ Feature Name } \\
\hline Temporal Feature & 1 & Zero Crossing Rate \\
\hline Statistical Features & 7 & $\begin{array}{l}\text { Mean, Min, Max, Standard Deviation, } \\
\text { Dynamic range, Crest Factor, Entropy }\end{array}$ \\
\hline Spectral Features & 9 & $\begin{array}{l}\text { Spectral Rolloff-85\% and 95\%, Spectra Spread, } \\
\text { Spectral Centroid, Flatness, Brightness, } \\
\text { Roughness, Irregularity, Kurtosis }\end{array}$ \\
\hline $\begin{array}{l}\text { Mel-frequency cepstral } \\
\text { coefficient (MFCC) }\end{array}$ & 13 & 1 to 13 coefficients \\
\hline
\end{tabular}

Category wise classification accuracy (\%) is shown in Figure 5.

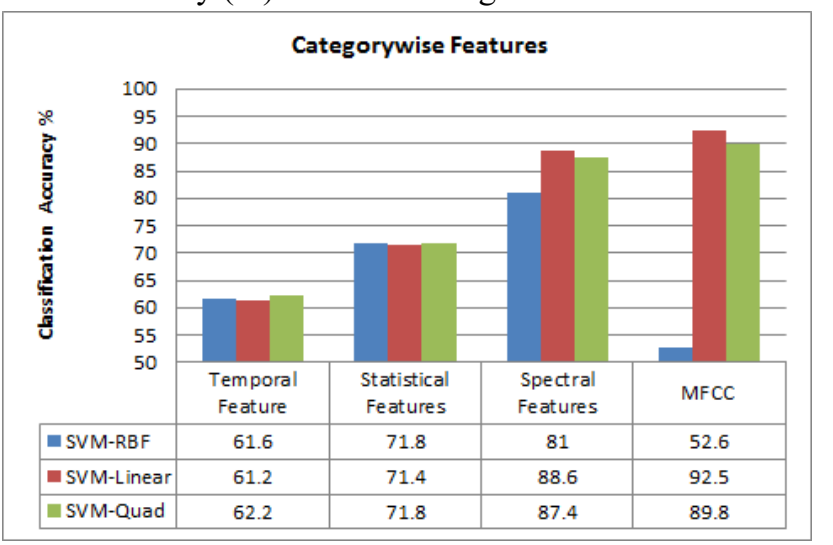

Fig. 5. SVM average accuracy $(\%)$ for category wise selected features 
MFCC has given highest accuracy $92.5 \%$ using SVM linear kernel

\section{Conclusion}

In this paper, three experiments were conducted to assess the impact of feature selection using filter approach using statistical methods such as MANOVA and Chi-square test and SVM kernels to classify Indian string instruments. Total 30 audio features including temporal, spectral, statistical and MFCC were extracted and used for experiments. Before classification, 25 of the 30 features are filtered, and only the top 5 are chosen Both methods have a diverse set of selected features. In MANOVA, features selected are Mean, Flatness,MFFCC 4th and 8th coefficient and standard deviation whereas in Chi-square, Mean, Flatness, Zero crossing rate, MFCC 4th coefficient and spectral rolloff with 85 frequency are selected as a prominent features. Similarly, without the MFCC 13 coefficients, 17 features are considered, and significant features are identified, notably, in MANOVA, mean, flatness, standard deviation, spectral spread, and spectral rolloff, and in Chi-square, mean, flatness, zero crossing rate, spectral rolloff, and standard deviation. SVM classifier was used to validate the impact of feature selection.

In experiment 1, the top five features with MFCC and SVM classifier using Linear kernel gave the highest accuracy of $93.4 \%$ and without MFCC $90.2 \%$. In experiment 2, the top 5 features with MFCC and SVM classifier with radial basis kernel achieve $93.4 \%$ highest accuracy, while the top 5 features without MFCC and SVM with linear kernel achieve $92.2 \%$ highest accuracy. In experiment 3 , a set of features is classified according to their type. The 13 MFCC coefficients achieve the highest accuracy of $92.5 \%$.

Although the results show that the filter approaches Chi-square and MANOVA have nearly the same accuracy for combining all top 5 features, the features ranked by chi-square are more significant than MANOVA. After examining the confusion matrix, it was discovered that Guitar and Santoor instruments are rarely misclassified with the remaining instruments, with the exception of Sitar, Sarod, and Veena, which have a high misclassification rate with each other.

\section{References}

[1] Ashwini and K. A. Vijaya(2020). Feature Selection For Indian Instrument Recognition Using SVM Classifier. International Conference on Intelligent Engineering and Management (ICIEM), pp. 277-280. DOI: 10.1109/ICIEM48762.2020.9160223.

[2] Bosch Juan J., Jordi Janer, Ferdinand Fuhrmann and Perfecto Herrera(2012). A Comparision of Sound Segregation Techniques for Predominant Instrument Recognition in Musical Audio Signals. International Society for Music Information RetrievalConference

[3] Bommert Andrea, Xudong Sun, Bernd Bischl, Jörg Rahnenführer, Michel Lang(2020). Benchmark for filter methods for feature selection in high-dimensional classification data. Computational Statistics \& Data Analysis,Volume 143. DOI: 10.1016/j.csda.2019.106839.

[4] Chandan S. V, M. R. Naik, Ashwini and A. V. Krishna(2019). Indian Instrument Identification from Polyphonic Audio using KNN Classifier. International Conference on Wireless Communications Signal Processing and Networking (WiSPNET), pp. 135-139. DOI: 10.1109/WiSPNET45539.2019. 9032860.

[5] Chen Shi-Huang, Chen Shih-Hao(2009). Content-based Music Genre Classification Using TimbralFeature Vectors and Support Vector Machine. ICIS,ACM

[6] Elaiyarajaa V., P. Meenakshi Sundaramb(2012). Audio Classification Using Support Vector Machines and Independent Component Analysis.Journal of Computer Applications (JCA),ISSN: 0974-1925, Volume V, Issue 1.

[7] Ghosal Arijit, Suchibrota Dutta and Debanjan Banerjee(2020). Classification of Speech and Song Using Co-occurrence-Based Approach. Computational Intelligence in Pattern Recognition,Advances in Intelligent Systems and Computing,Springer Nature,pp138-146. DOI- 10.1007/978-981-13-9042-5_11

[8] Joder Cyril, Slim Essid, and Gaël Richard(2009). Temporal Integration for Audio Classification With Application to Musical Instrument Classification. IEEE Transactions on Audio, Speech and Language Processing, vol. 17, NO. 1.

[9] Joshi Swarupa, Chitre Abhijit(2015). Identification of Indian Musical Instruments by Feature Analysis with Different Classifiers. International Conference on Computer and Communication Technology,ACM, pp 110-114. DOI-10.1145/2818567.2818588

[10] Kakarwal Sangeeta, Chaudhary Seema R(2020). Analysis of Musical String Instruments using k-NN.IEEE International Conference on Smart Innovations in Design, Environment, Management, Planning and Computing

[11] Kothe R.S, D.G.Bhalke,P.P.Gutal.Musical(2016). Instrument Recognition using K-Nearest Neighbour and Support Vector Machine.IEEE International Conference on Advances in Electronics, Communication and Computer Technology (ICAECCT).

[12] Kumar A., S. S. Solanki and M. Chandra(2020). Study of Indian instruments for designing a speech/music classifier, 5th International Conference on Computing, Communication and Security (ICCCS),pp. 1-7, DOI: 10.1109/ICCCS49678.2020.9276825.

[13] Liu J. and L. Xie (2010). SVM-Based Automatic Classification of Musical Instruments. International Conference on Intelligent Computation Technology and Automation, pp. 669-673, DOI: 10.1109/ICICTA.2010.64.

[14] McHugh Mary L(2013). The Chi-square test of independence.,DOI:10.11613/BM.2013.018.

[15] Ozerov A., E. Vincent, and F. Bimbot(2013). A General Flexible Framework for the Handling of Prior Information in Audio Source Separation. IEEE Transactions on Audio, Speech and Language Processing, vol. 20, no. 4, pp. $1118-1133$.

[16] Peetersa Geoffroy, Bruno L. Giordano, Patrick Susini and Nicolas Misdariis, Stephen McAdams(2011). The Timbre Toolbox: Extracting audio descriptors from musical Signals.J. Acoustical Society of America.

[17] Racharla Karthikeya, Vineet Kumar, Chaudhari Bhushan Jayant, Ankit Khairkar and Paturu Harish(2020), Predominant. Musical Instrument Classification based on Spectral Features. IEEE International Conference on Signal Processing and Integrated Networks (SPIN) 
[18] Sangeetha Rajesh and. Nalini N. J(2020). Singer Identification Using MFCC and CRP Features with Support Vector Machines. Computational Intelligence in Pattern Recognition,Advances in Intelligent Systems and Computing,Springer Nature,pp. 306-317. DOI- 10.1007/978-981-13-9042-5 25

[19] Shetty Surendra and Hegde Sarika(2020). Automatic Classification of Carnatic Music Instruments Using MFCC and LPC. Data Management, Analytics and Innovation, Advances in Intelligent Systems and Computing,Springer Nature Singapore, pp. 458-469 . DOI- 10.1007/978-981-32-9949-8 32

[20] Steyn H. S. Jr. \& S. M. Ellis[2009]. Estimating an Effect Size in One-Way Multivariate Analysis of Variance (MANOVA) Multivariate Behavioral Research, 44:1, 106-129, DOI:10.1080/00273170802620238

[21] Vinutha T. P., Suryanarayana Sankagiri and Preeti Rao(2016). Reliable Tempo Detection for Structural Segmentation in Sarod Concerts,Twenty Second National Conference on Communication (NCC) ,IEEE..DOI- 10.1109/NCC.2016.7561163

\section{Author Profile}
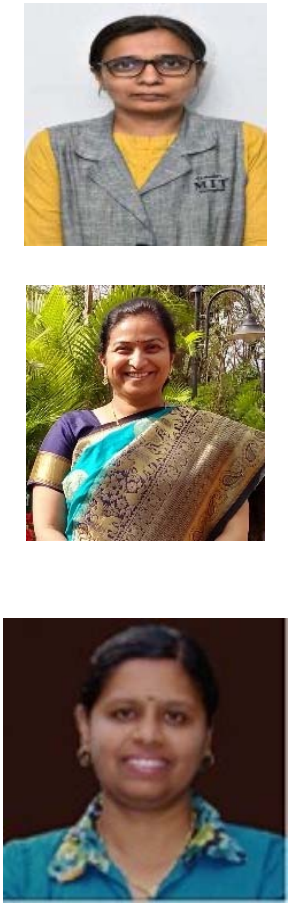

Seema R. Chaudhary is presently working as a Associate Professor in CSE dept at Maharashtra Institute of Technology,Aurangabad(MS), India. She is pursuing Ph.D from Dr.B.A.Marathwada University, India. Her main areas of interest is audio signal processing, image processing, data mining and software development

Dr. Sangeeta N. Kakarwalis working as a Associate Professor in Dept. of Computer Science and Engineering, Aurangabad(MS),India. Her area of specialization is pattern recognition and image processing

Dr. Jayashri V. Bagade is a Associate Professor in Information Technology Department at Vishwakarma Institute of Information Technology, Pune (India). She has graduated from BAMU University, Aurangabad, India in 2005 with Master of Engineering in Computer Science and Engineering and $\mathrm{PhD}$ in Computer Science and Engineering from Thapar Institute of Engineering \& Technology, Patiala, Punjab (India).Her main areas of interest are Information Storage and Retrieval, Image Processing and Soft Computing. She is reviewer of reputed journal in India and abroad. 